\title{
Flexible platform with wireless interface for DC-motor remote control
}

\author{
G. Litta, R. Di Rienzo, R. Morello, R. Roncella, F. Baronti, and R. Saletti \\ Dip. Ingegneria dell'Informazione, Università di Pisa, Via Caruso 16, Pisa, Italy
}

\begin{abstract}
Several portable applications, such as small electric vehicles and power tools, often require the use of direct current (DC) motors that significantly differ from one to another in terms of power, torque, and driving techniques. New market requirements of these applications suggest the implementation of smart user interfaces that may allow the introduction of those devices in the new Internet of Things paradigm by making them connected. This paper discusses the design and verification of a flexible platform able to drive different types of DC motors that is also provided with a Bluetooth connection for remote control and monitoring. As the platform can drive different motors with different driving techniques, it provides standardisation and cost reduction in the production of a set of tools. Two gardening tools are used as case study to verify the design and flexibility of the board. Both tools are successfully controlled and monitored with a wireless connected remote user interface.
\end{abstract}

\section{INTRODUCTION}

The number of portable applications in which electric DC motors are used has considerably grown in the last years. Good examples are the green electric mobility field, where e-bikes, e-scooters and other small vehicles have been introduced, the home automation field and the power tools used for building and gardening applications. The above mentioned application fields require motors with very different mechanical, electrical and economical characteristics. These requirements can only be satisfied by using different types of motor for each particular application. The most common types of motors are the brushed DC (BDC) and brushless DC (BLDC) motors. The first is less expensive and heavier than the second one, which instead is more efficient, quiet and reliable [1]. Moreover, a BDC motor only needs two power contacts and it is simpler to control, because it starts to spin as soon as power is applied to it. Instead, the BLDC motor has more than two power contacts and needs a driver that provides the specific sequence of control signals necessary for the correct operation. Both types of motors are typically controlled by an electronic board that provides the power signals and manages the motor utilization in the application, e.g. by controlling its speed or torque [2]. Many solutions have been presented in the literature to deal with this task. Usually, motor control boards are composed of two parts: the first satisfies the application needs, counting on the flexibility of a general purpose microcontroller that executes the required application algorithms. The second one usually generates the power signals needed to drive the motor. It contains semiconductor power switches and a digital signal processor (DSP) which offers the computational power needed by the motor control techniques employed [3], [4]. This

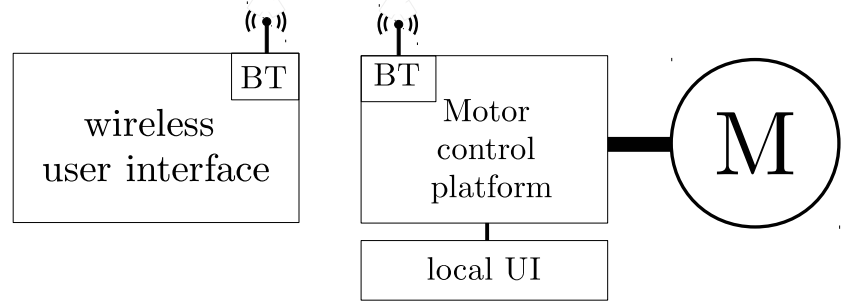

Fig. 1. Block diagram of the remotely controlled motor driver platform application.

solution achieves great performance, but it is expensive and not flexible, so it is mainly used in particular applications. A good trade-off between performance and cost can be reached by using a single CPU unit, usually a microcontroller with medium/high performance, which simplifies the control board and increases its reliability.

The motor control system is often equipped with a simple user interface (UI) that allows the user to control the motor, by setting a very limited number of parameters and operating conditions. Many advantages can be achieved if a remote interface, which complies to the new Internet of Things (IoT) paradigm [5], is added to the local UI to manage the motor control system. The complexity of the remote control features depends on the application, but the basic idea is that users are enabled to completely control the system in any moment and from any place by using a smart device with a wireless connection, such as a PC, a tablet or a smartphone. For example, this remote control approach would be very useful with motors located in places not easily accessible, or where the wiring introduces issues of cost, complexity and reliability. A possible solution is to provide the motor control board with a Wi-Fi module that supports the TCP/IP stack. This approach allows the platform to be controlled by means of a pointto-point connection [6] or a device connected to the Internet [7]. In applications where a point-to-point connection only is sufficient, a Bluetooth interface provides a more simple, lowcost, and low-energy communication link than the Wi-Fi one [8]. In fact, the power needed for communication is strongly reduced if a Smart Bluetooth interface is adopted.

This paper discusses the design and the realization of a flexible platform that enables the drive and the remote control of different types of DC motors. The platform behaviour is 


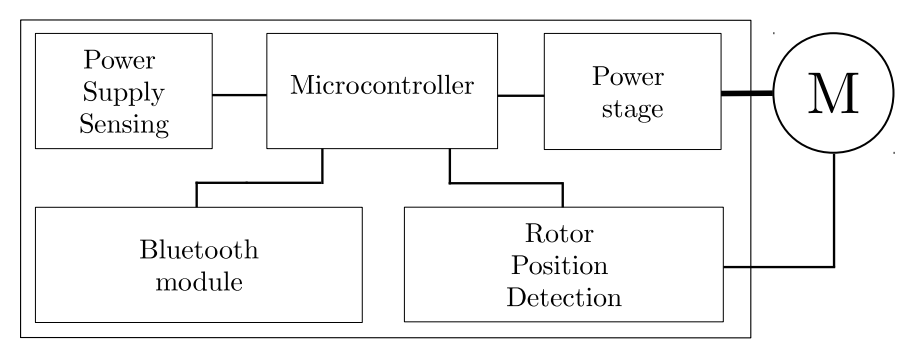

Fig. 2. Motor control platform block diagram.

controlled through a wireless connection interface. In this way, the same platform can be used in a wide range of applications that differ in the type of motor adopted and the control strategy implemented. This feature is very interesting when a new device is going to be developed, because the platform can be reused with minor adjustments and with a considerable reduction of the development costs. Moreover, the platform allows the remote monitor/control of the connected motor by making it available to the user according to the IoT scenario. The platform architecture is shown in Fig. 11. It is based on a single microcontroller and a Bluetooth interface that lead to a good trade-off between flexibility, performance and cost.

The paper is organised as follows. The next Section describes the proposed platform in details. Section III describes a case study used to demonstrate the operation of the platform. The experimental results are shown in Section $[\mathrm{IV}$ and finally some conclusions are drawn in Section $\mathrm{V}$.

\section{Wireless Motor CONTROL PLATFORM}

The block diagram of the motor control platform is shown in Fig. 2. The core of the platform is the Microcontroller, the main function of which is to drive the motor by feeding the Power stage. Appropriate signals are generated according to the type of the used motor and to the information acquired from the Rotor Position Detection and the Power Supply Sensing blocks. The platform is monitored and controlled by means of a wireless user interface connected via Bluetooth. The motor control strategies, the control parameters and the motor type can be uploaded to the platform making it flexible and usable with various kinds of motor and size. The entire system is described in more detail in the following subsections.

\section{A. Microcontroller}

An NXP Semiconductor LPC1549 microcontroller based on a 32-bit ARM cortex-M3 CPU is used in the platform described here. It is equipped with a State Configurable Timer (SCTimer/PWM) peripheral that is very useful for motor control [9]. It is a multi-purpose state configurable timer, which allows the generation of the signals that drive the power stage. This is possible thanks to a state machine that changes its state according to the values of the signals provided by the rotor position detection block. The state machine is implemented according to the typology of the driven motor. For BDC motors, it allows the regulation of the speed and the direction of the rotation, whereas more complex driving strategies are used to drive brushless motors. In BLDC motors, the motor rotation is determined by the interaction between the stator magnetic field and the rotor one, which is due to the permanent magnets located in it. The stator rotating magnetic field is generated by the currents flowing in the stator coils. A BLDC driving technique is used to properly drive the motor. Two different methods are usually adopted: the vectorial or the scalar control. In the vectorial control method, the stator current is controlled in the $\mathrm{d}-\mathrm{q}$ vector space by performing vectorial operations [10]. An accurate control of the magnetic flux is guaranteed at the expense of a high computational burden. The scalar technique is less expensive than the previous one in term of processing power. In fact, the stator magnetic field is only computed for a finite number of rotor positions, reducing the mathematical operations to be executed. Both techniques can be implemented in the above mentioned SCTimer/PWM peripheral. The outputs of this peripheral are Pulse Width Modulation (PWM) signals. They drive the semiconductor power switches located in the power stage, by which the motor windings are supplied.

The use of a dedicated control peripheral reduces the CPU computational load for driving the motor and allows the microcontroller to execute other tasks, such as communicating with the wireless interface. Each task is developed in software as a thread scheduled by the FreeRTOS operating system running on the CPU. It is a real-time operating system for embedded architectures. The data exchange between the running tasks is achieved by sharing blocks of memory.

The main task executed by the CPU is dedicated to control the motor behaviour. The driving strategy changes according to the type of motor that is used in the particular application. In any case, this task consists of two parts: a state machine used to configure the SCTimer/PWM peripheral with the driving data provided by the user, and a standard ProportionalIntegrative-Derivative (PID) controller. The latter is used as speed or torque regulator that changes the duty-cycle of the PWM signals used to drive the motor in a closed loop. The three characteristic gains of the PID regulator, i.e., $K_{P}, K_{I}$, and $K_{D}$, can be calculated by using the classic Ziegler-Nichols (Z-N) tuning method or other empirical ones [11]. The proper control parameters for the particular motor driven can be uploaded to the platform via the Bluetooth interface.

\section{B. Power stage}

The power stage receives the PWM signals coming from the microcontroller and provides the supply to the motor windings with a power compatible with the motor used in the application. It is composed by a N-MOS three-phase inverter and the gate driver DVR8301 by Texas Instrument. The DVR8301 is a very powerful chip that drives the MOSFETs of the inverter and preserves the system integrity in case of malfunctions, with built-in protection circuits. In fact, this chip applies a mechanism to prevent the short circuit on the power supply that may occur when a low-side and a high-side MOSFETs are simultaneously switched. At the same time, it provides an integrated sensing of the $V_{D S}$ of each MOSFET in order to 
protect the power stage in case of overcurrents. All the three legs of the inverter are utilized to drive 3-phases brushless motors, whereas only two legs are used (i.e., a H-bridge configuration) with BDC motors. The DVR8301 operates up to $60 \mathrm{~V}$, a value compatible to many applications in home automation and gardening.

\section{Rotor Position Detection}

The knowledge of the rotor position of the motor allows the calculation of its rotational speed [12]. Usually, the rotor position is detected by using Hall sensors or optical encoders that provide the microcontroller with digital signals related to the rotor position. The microcontroller calculates the motor speed by measuring the period of the position signals coming from the position sensors. The knowledge of the rotor position is also mandatory for the appropriate generation of the stator magnetic field in BLDC motors. In some applications, the rotor position is detected without sensors to reduce the motor cost and wiring. It is possible by using the current and voltage measured on the motor phases. For example, a sensorless application with zero crossing detection technique is described in [13]. The zero crossing point of the back electromotive force (BEMF) induced on the stator coil is detected when the coil is not powered. If the procedure is repeated on the three coils, it is possible to generate three signals that reproduce the response of the rotor position sensors. This technique is applied in the platform described here, by using the circuit presented in [14]. Therefore, the flexibility of the motor control platform is further improved, as it is able to manage both BLDC motors, with and without sensors.

\section{Power supply sensing block}

The power supply sensing block allows the microcontroller to measure the board supply voltage and current. This feature is useful to determine the power consumption of the system in the various operation phases. A dedicated software task acquires these data by using two channels of the analog-todigital converter (ADC) of the microcontroller.

\section{E. Local user interface}

The board is equipped with a very basic local interface, composed of one LED and five push buttons. The developer can program the functions of the UI according to its own needs. For example, the buttons can be used to activate the motor, to control the rotational speed, and to enable the visualization of the motor status on the LED.

\section{F. Bluetooth module}

The point-to-point communication link between the board and the external user is established by the Bluetooth lowenergy (BLE) module BGM111 by Silicon Labs. This BLE module uses Bluetooth Smart, a new type of protocol that presents some differences with respect to the classic Bluetooth. Bluetooth Smart devices are designed for intermittent transfers of data and do not include the Serial Port Profile (SPP) that emulates a serial port, as it is available in standard Bluetooth

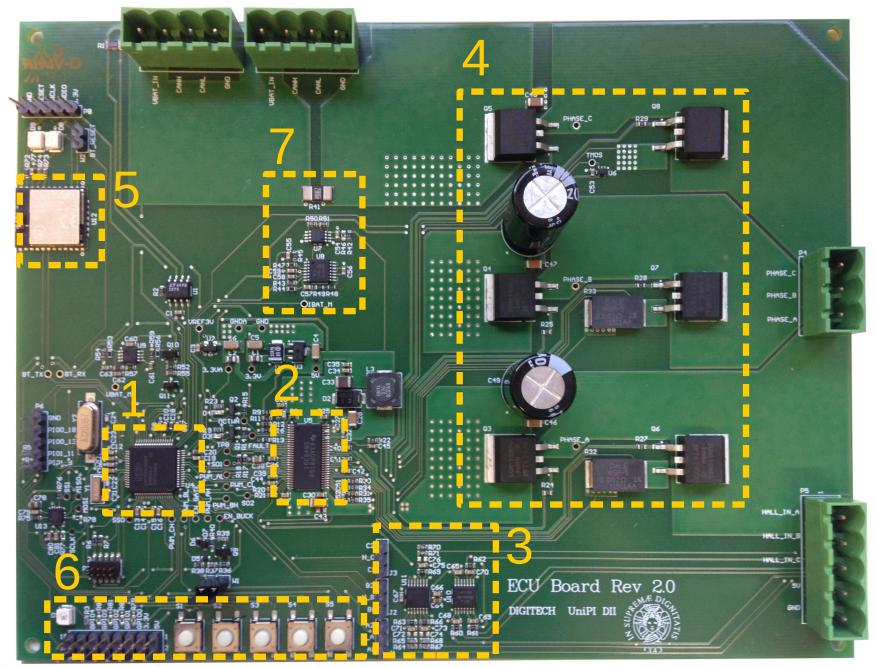

Fig. 3. Photograph of the implemented platform. The main blocks are: 1 . Main unit, 2. Gate Driver, 3. Rotor Position Detection, 4. Inverter, and 5. Bluetooth module, 6. local user interface, 7. sensing block

devices [15]. A custom protocol compliant with the Bluetooth Smart paradigm has been developed in order to realize a serial communication link on the BLE module. It is based on the generic attribute profile (GATT) that defines a hierarchical data structure shared by the BLE devices involved in the communication.

The developed application runs on the BLE module and consists of two parts. The first one uses the standard service of the protocol to make the device visible by showing its name. Instead, the other part implements the communication through a custom service which emulates a SPP. The BLE module receives the data from the microcontroller over a UART interface, creates a communication packet and sends it to the connected device. The packet contains a preamble with an identification code and the payload which contains the useful data. The same service also manages the data received from the connected device, implementing a server. The user remotely controls the motor by means of a wireless UI. The wireless UI is a client application that, after the connection with the server is established, is able to exchange data over the wireless link. In particular, it applies the custom protocol with an identification code by which the information is encoded and decoded.

\section{CASE STUDY}

The motor control platform with wireless connection described here has been realized with a double-layer PCB, as shown in Fig. 3, where the main components described in the previous Section are highlighted. The platform has been used to control the motors of a set of battery powered tools for gardening applications. Every tool of the set is powered by the same battery, but each one presents different power requirements, also because of the different types of motors used. A production line based on this idea, i.e., the use of the same flexible platform in each tool, may enjoy a 


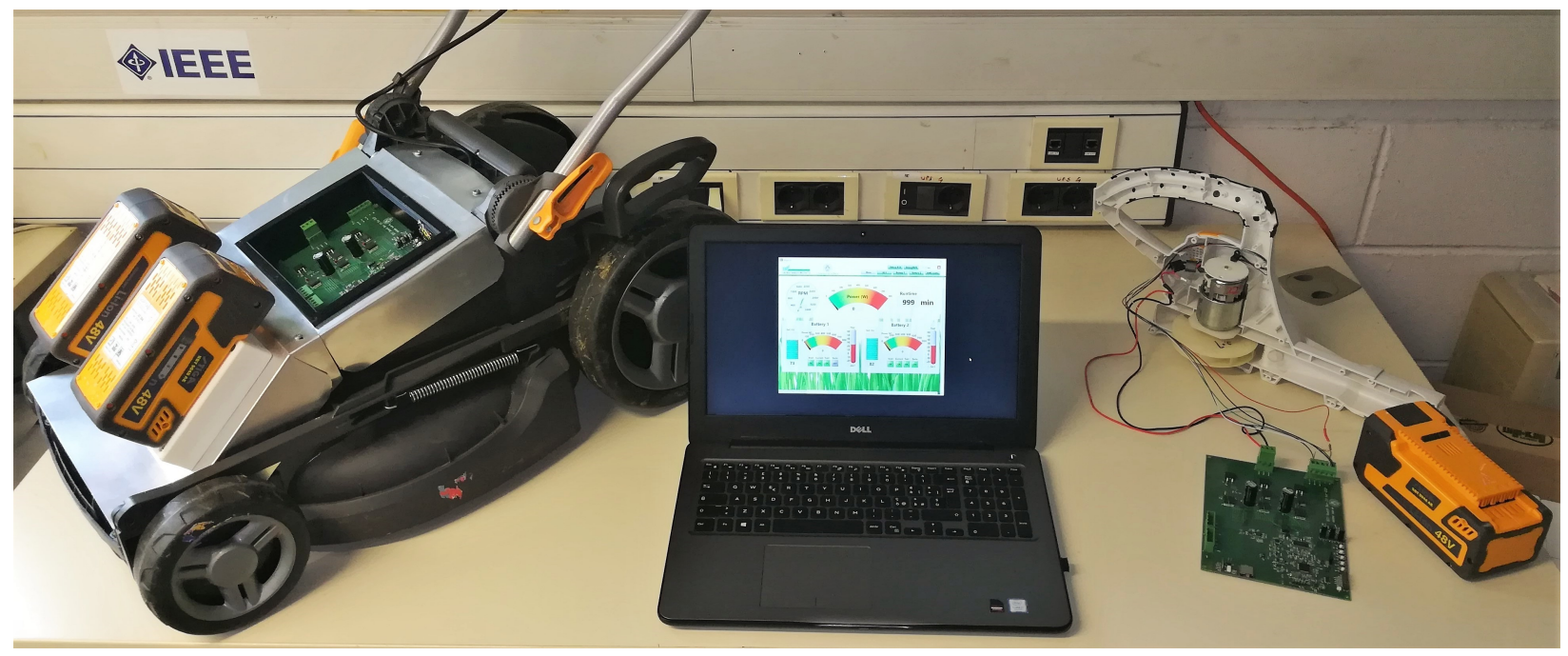

Fig. 4. Photograph of the motor control platform used in the lawnmower and in the blower and the relative wireless user interface.

significant reduction of the costs. This is due to the lower cost in the development of the hardware devices and the firmware, but also to the reduction of the production costs of the motor controller board, which is shared among the tools. In particular, we selected two tools, a lawnmower and a blower, shown in Fig. 4 Both are controlled by the same wireless UI which has been developed in LabVIEW and executed on a remote PC. The use of a single UI application that is applicable to the entire set of tools brings to a further reduction of the development and maintenance costs. The platform is programmed to control the power provided to the motors by implementing a closed-loop technique which maintains a constant motor speed, as remotely set by the user. At the same time, the tool behaviour is remotely monitored by showing the motor status and parameters on the UI.

The lawnmower has a $48 \mathrm{~V}, 400 \mathrm{~W}, 3$-phase BLDC motor with embedded Hall sensors. However, to demonstrate the flexibility of the platform, the sensorless technique described in Section II-C was applied to measure the rotor position, by disconnecting the Hall sensors. The microcontroller implements the scalar six-step driving strategy [16], which powers the stator coils of the motor in six different steps that are periodically repeated with a sequence depending on the rotor position. The speed of this tool can be set in a range from $1500 \mathrm{RPM}$ up to $3600 \mathrm{RPM}$.

Instead, the blower has a $48 \mathrm{~V}, 250 \mathrm{~W}$ BDC motor. An optical encoder is used to measure the speed of the motor that can reach a value of about $11000 \mathrm{RPM}$.

The tools are remotely controlled with the wireless UI by sending commands to the platform that set some system parameters, such as the reference speed. At the same time, the motor behaviour is remotely monitored on the same wireless UI. The remote interface can be adapted for any device provided with the Smart Bluetooth protocol, such as smartphones and tablets. The use of the interface on a portable device brings many advantages. In fact, it can be used for the online visualization of useful information such as the instant speed of the motor and its power consumption by using graphical indicators. As these gardening tools are equipped with smart batteries, the UI is also capable of showing the state of the battery, in terms of voltage, current and residual charge. Furthermore, it creates a log file where all the data received from the platform are stored, allowing the user to analyse the behaviour and the performance of the tools.

\section{EXPERIMENTAL RESULTS}

Various experimental tests have been performed on both tools in order to verify the correct functionality of the presented platform. The wireless UI acts as a remote monitor/control device, as it stores the information acquired from the tools during the tests, and allows the user to set different motor speed profiles.

In the first test reported, we evaluate the behaviour of the entire system and the effectiveness of the control algorithms by performing off-load measurements of the motor speed. To this end, the speed is set by stepping its value up to the maximum allowed by the application and then returning back again. The comparison between the measured and the reference speed values is shown in Fig. 5 for both the tools under test. We note that the controller behaviour is correct, as it changes the motor speed according to the value set by the user. However, Fig. 5(a) also shows that the two speed values present a noticeable difference in the transient when the lawnmower motor speed is asked to step down. This is ascribed to the slow down policy implemented in the controller, which reduces the power provided to the motor without applying any breaking procedure. The large inertia of the cutting blade attached to the motor free wheeling does not allow the speed to quickly slow down and reach the set value. This effect does not occur in such an evident way in the blower, because of the smaller inertia of its fan, as we can see in Fig. 5(b) 


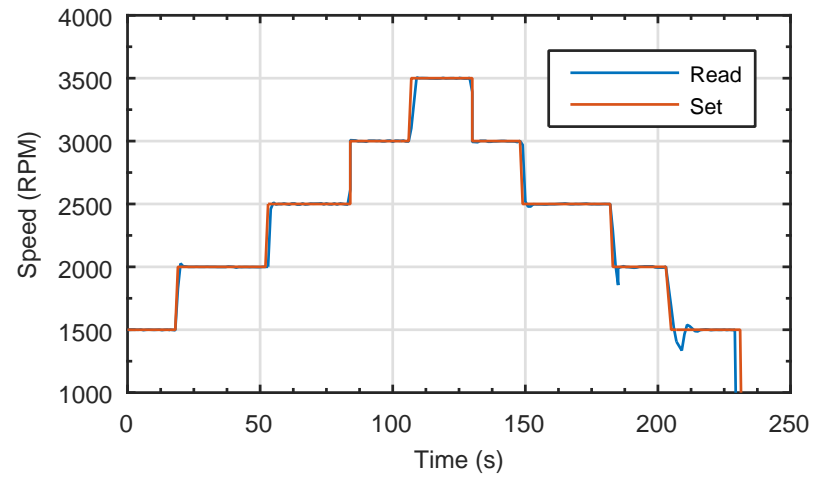

(a)

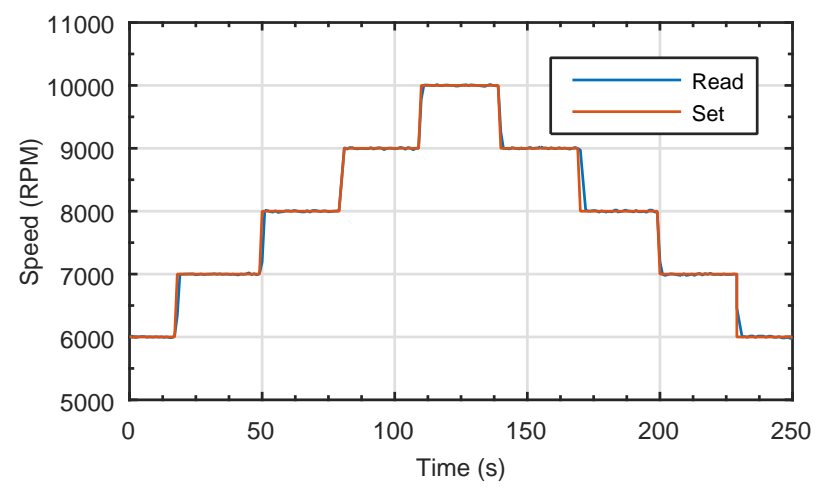

(b)

Fig. 5. Comparison between the read and set speeds acquired on the lawnmower (a) and on the blower (b).

In order to verify the capability of the motor controller to keep the speed fixed to the set value, tests on the working field of the tools have also been performed. Significant variations of the motor load can be appreciated on the lawnmower during the grass-cutting process, due to the uneven surface of the grass. The situation is completely different for the blower that usually presents an almost constant load. Fig. 6 shows the speed variations of the lawnmower motor during two minutes of work. The target speed is set to the reference value of $3500 \mathrm{RPM}$, but the instantaneous speed of the motor changes because of the variable resistance offered by the grass. The controller counteracts these variations by changing the power provided to the motor: when the speed decreases the controller gives more power to the motor and vice versa. This behaviour is illustrated in Fig. 6, which shows the variations of the instantaneous power calculated by using the quantities acquired by the sensing block.

\section{CONCLUSIONS}

This paper has discussed the design and the verification of a flexible DC motor control platform provided with a wireless connection to a remote interface. The main feature of the platform is its capability of driving different types of DC motors with the same hardware structure. BDC and BLDC motors can be driven with various driving techniques, either scalar or vectorial control, and sensored or sensorless rotor position

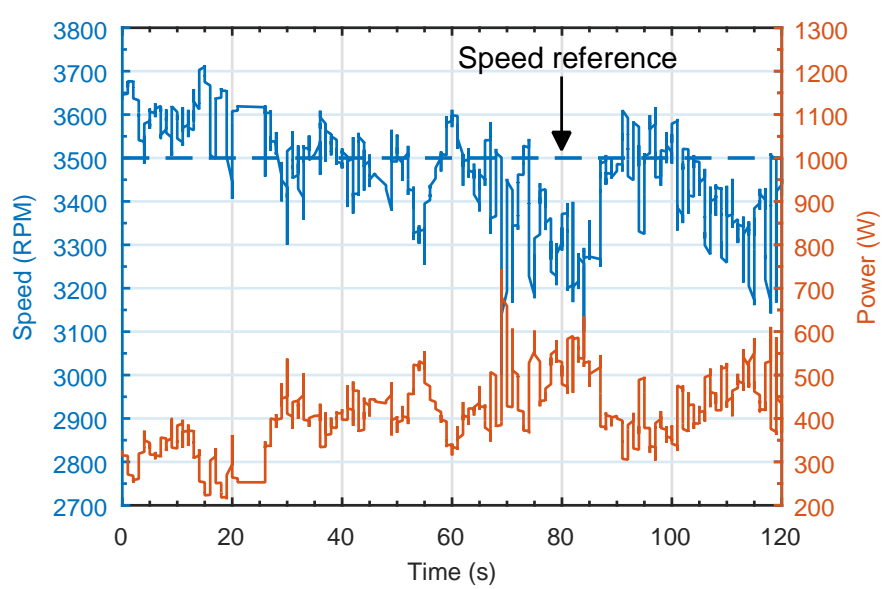

Fig. 6. Motor speed and instant power consumption of the lawnmower at work.

detection. The wireless link enables the remote connection to the platform. It makes it possible to tailor the platform to the typology of the motor to be driven, by even changing the parameters of the driving control algorithms. Moreover, the remote interface that runs as software application on any smartphone and tablet provided with a BLE connection makes it possible to both monitor and control the DC motor. The speed or torque of the motor can remotely be set and the motor parameters and status can be seen with graphical indications on the interface, thus introducing in the IoT scenario the system in which the motor is used.

A case study has been described, in which the platform has been applied to drive the motors of different battery powered gardening tools. Two tools with different motor types, different rotation speeds, and different load profiles have been investigated, when powered by the flexible and connected platform. The experiments show that the platform correctly drives the motors in the two applications by remotely controlling the motor speeds. Moreover, the monitoring capability of the connected platform allows us to remotely collect the data coming from the applications and analyse the performance of the motor and the controller algorithm. The experimental results show that the same flexible platform can be utilized to drive various DC motors in applications that may significantly differ to each other, leading to hardware standardization and cost reductions in the development of the devices.

\section{REFERENCES}

[1] S. Derammelaere, M. Haemers, J. D. Viaene, F. Verbelen, and K. Stockman, "A quantitative comparison between BLDC, PMSM, brushed DC and stepping motor technologies," in 2016 19th International Conference on Electrical Machines and Systems (ICEMS), Nov 2016, pp. 1-5.

[2] C. Bates, "The principles of electronic motor control," Students Quarterly Journal, vol. 22, no. 87, p. 99, 1952.

[3] M. A. Hassan, A. R. Abdullah, N. Bahari, and A. Jidin, "Incorporating brushless DC motor in outdoor fan control of low voltage airconditioning system," in 2013 IEEE Student Conference on Research and Developement. IEEE, Dec 2013, pp. 550-555.

[4] H.-C. Wu, M.-Y. Wen, and C.-C. Wong, "Speed control of BLDC motors using hall effect sensors based on DSP," in 2016 International Conference on System Science and Engineering (ICSSE). IEEE, Jul 2016, pp. 1-4. 
[5] A. H. de Sousa, A. T. Y. Watanabe, J. de Oliveira, L. R. Lima, and B. H. K. de Paula, "Monitoring, Controlling and Configuring of Wireless Household-Electric Network through Remote Virtual Interface," in 2007 5th IEEE International Conference on Industrial Informatics. IEEE, Jul 2007, pp. 51-56.

[6] D. Sarb and R. Bogdan, "Wireless motor control in automotive industry," in 2016 24th Telecommunications Forum (TELFOR). IEEE, Nov 2016, pp. 1-4.

[7] C.-T. Chu, H.-K. Chiang, and Y.-S. Chang, "Wireless cloud interaction system implement motor control," in 2016 11th International Microsystems, Packaging, Assembly and Circuits Technology Conference (IMPACT). IEEE, Oct 2016, pp. 325-328.

[8] S. Das, S. Ganguly, S. Ghosh, R. Sarker, and D. Sengupta, "A bluetooth based sophisticated home automation system using smartphone," in 2016 International Conference on Intelligent Control Power and Instrumentation (ICICPI). IEEE, Oct 2016, pp. 236-240.

[9] NXP Semicondutor, "AN11538 SCTimer/PWM Cookbook," Tech. Rep., 2016.

[10] L. Zhou, W. Gruber, and D. L. Trumper, "Position control for hysteresis Motors: A field-oriented control approach," in 2017 IEEE International Electric Machines and Drives Conference (IEMDC). IEEE, May 2017, pp. 1-8.

[11] P. M. Meshram and R. G. Kanojiya, "Tuning of PID controller using Ziegler-Nichols method for speed control of DC motor," in IEEEInternational Conference On Advances In Engineering, Science And Management (ICAESM -2012), 2012, pp. 117-122.
[12] M. Baszynski and S. Pirog, "A Novel Speed Measurement Method for a High-Speed BLDC Motor Based on the Signals From the Rotor Position Sensor," IEEE Transactions on Industrial Informatics, vol. 10, no. 1, pp. 84-91, Feb 2014.

[13] X. Zhang, H. Gao, and C. Zeng, "Rotor position detection based on BEMF zero-crossing detection method with three-EMF force superposition," in 2016 13th International Conference on Ubiquitous Robots and Ambient Intelligence (URAI). IEEE, Aug 2016, pp. 740-745.

[14] T. Kim, Chungil Kim, and J. Lyou, "A new sensorless drive scheme for a BLDC motor based on the terminal voltage difference," in IECON 2011 - 37th Annual Conference of the IEEE Industrial Electronics Society. IEEE, Nov 2011, pp. 1710-1715.

[15] I. Horvat, N. Lukac, R. Pavlovic, and D. Starcevic, "Smart plug solution based on bluetooth low energy," in 2015 IEEE 5th International Conference on Consumer Electronics - Berlin (ICCE-Berlin). IEEE, Sep 2015, pp. 435-437.

[16] E. Firmansyah, F. D. Wijaya, W. P. R. Aditya, and R. Wicaksono, "Six-step commutation with round robin state machine to alleviate error in hall-effect-sensor reading for BLDC motor control," in 2014 International Conference on Electrical Engineering and Computer Science (ICEECS). IEEE, Nov 2014, pp. 251-253. 